Meta

Journal des traducteurs

Translators' Journal

\title{
Sur le bitume
}

\section{Henri Dumas}

Volume 20, numéro 3, septembre 1975

URI : https://id.erudit.org/iderudit/002673ar

DOI : https://doi.org/10.7202/002673ar

Aller au sommaire du numéro

Éditeur(s)

Les Presses de l'Université de Montréal

ISSN

0026-0452 (imprimé)

1492-1421 (numérique)

Découvrir la revue

Citer cet article

Dumas, H. (1975). Sur le bitume. Meta, 20(3), 208-217.

https://doi.org/10.7202/002673ar

Ce document est protégé par la loi sur le droit d'auteur. L’utilisation des services d'Érudit (y compris la reproduction) est assujettie à sa politique d'utilisation que vous pouvez consulter en ligne.

https://apropos.erudit.org/fr/usagers/politique-dutilisation/
Cet article est diffusé et préservé par Érudit.

Érudit est un consortium interuniversitaire sans but lucratif composé de l’Université de Montréal, l'Université Laval et l'Université du Québec à Montréal. Il a pour mission la promotion et la valorisation de la recherche. https://www.erudit.org/fr/ 


\section{SUR LE BITUME}

"Et là, comme une brute, dans le dos Il lui planta son grand couteau. Elle tomba sur le bitume, sans dire un seul mot."

(La rime est moins classique que le procédé.) Ami traducteur, te souviens-tu de cette vieille complainte des boulevards (du Crime, entre autres) ? Sur le bitume, manquons-nous de mots? Non; nous allons voir que nous en avons, peut-être même, trop. Le hasard, qui nous a déjà si bien aidé ${ }^{1}$, et qui a bien mérité sa promotion au rang de providence, a guidé notre démarche - notre flânerie, si tu veux - le long des avenues de la terminologie, vers une précieuse source bitumineuse : les Chaussées modernes, par P. Le Gavrian.

1. «Les jeux du hasard ", l'Actualité terminologique, vol. 6, $\mathrm{n}^{\circ}$ 8, octobre 1973. 
Cet ouvrage date, certes (la deuxième édition que nous avons en main est de 1934), mais nous nous intéressons aux mots et non aux techniques et cela nous chaut peu. En outre, les produits bitumineux sont vieux comme l'Arche de Noé; d'ailleurs, en cette première moitié du siècle, on avait encore souci de la précision du langage, de la constance du vocabulaire et de sa défense contre les diverses pollutions. En particulier, l'usage des termes les plus courants encore aujourd'hui avait déjà été normalisé.

Pour la terminologie française, la situation est très claire. Le français est en effet allergique à toute forme d'ambiguïté ou d'imprécision, dont il se débarrasse rapidement, soit par la voie naturelle - consensus des usagers de la langue - , soit, au besoin, par voie d'intervention normative. Langue de lumière qui, traitant de goudrons, brais et bitumes, reste limpide comme eau de roche! Mais le plus simple et le plus expéditif est encore de citer très largement l'ouvrage mentionné ci-dessus.

TERMINOLOGIE FRANÇAISE. Il faut signaler tout d'abord, la question des goudrons étant provisoirement laissée de côté, que le langage commercial usuel en France, établit pour les termes bitume et asphalte des distinctions assez nettes, mais qui ne concordent ni avec la doctrine scientifique, ni avec l'étymologie, ni avec les définitions étrangères rappelées ci-dessus.

On a coutume, en France, dans les milieux des ingénieurs et des entrepreneurs, d'appeler bitumes les seuls mélanges solides ou visqueux d'hydrocarbures provenant de la distillation des pétroles, ayant un pouvoir agglomérant ou adhésif, opaques, noirâtres ou bruns, solubles dans le sulfure de carbone et contenant peu de produits volatils ; en réalité, le mot français «bitume » est ici synonyme du mot américain asphalt. (ouvrage cité, p. 42)

Du côté anglais, nous avons à tenir compte des différences entre deux langues trop voisines pour qu'on n'oublie jamais qu'elles sont différentes, et trop séparées pour que cet oubli persistant soit sans danger. En fait, dans le cas de notre propos, la seule difficulté réelle vient d'asphalt, qui, en américain, correspond à « bitume», alors qu'en anglais il est l'équivalent d'« asphalte ».

Nous présentons ci-dessous une liste de termes équivalents, tirés de l'ouvrage cité et du Dictionnaire technique routier en 6 langues, de l'Association internationale permanente des congrès de la route. La présentation de cette liste surprendra certains; le but visé a été de réduire le temps de consultation au strict minimum compatible avec la sûreté de l'information. Il serait intéressant de connaître les réactions des utilisateurs, les traducteurs, devant cette nouvelle présentation.

01 asphalt (américain)

bitume

Matière solide ou semi-solide, de couleur noire ou brun foncé, qui se liquéfie graduellement par la chaleur et dont les constituants prédominants sont des bitumes, lesquels se rencontrent dans la nature à l'état solide ou semi-solide, ou sont obtenus en raffinant le pétrole, ou résultent de la combinaison desdits bitumes, soit entre eux, soit avec des pétroles ou leurs dérivés. 
02 asphalt (anglais)

asphalte

Produit naturel ou composé, dans lequel du bitume asphaltique sert

d'agglomérant à des matières minérales inertes. La dénomination

«asphalte» sera complétée par l'indication de l'origine du produit.

03 asphalte

asphalt

Cf. .02

04 asphalte brut

crude asphalt

Cf. 35

05 asphalte comprimé

compressed asphalt

Cf. 34

06 asphalte coulé

Cf. 63

07 asphalte en roche

native asphalt

rock asphalt (1)

Cf. 65

08 asphalte épuré

refined asphalt

Cf. 75

09 asphalte naturel native asphalt rock asphalt (1)

Cf. 65

10 asphaltene

asphaltène

Éléments d'un bitume asphaltique, soluble dans le sulfure de carbone et dans le tétrachlorure de carbone, mais insoluble dans l'huile de naphte normale.

11 asphaltène

asphaltene

Cf. 10

12 asphalte raffiné

refined asphalt

Cf. 12

13 asphaltic bitumen

bitume asphaltique

Bitume natif ou naturel ou bitume préparé à partir d'hydrocarbures 
naturels, ou leurs dérivés naturels obtenus par distillation, oxydation ou craquage ; solide ou peu fluide, contenant peu de produits volatils, ayant des propriétés agglomérantes caractéristiques, et pratiquement soluble dans le sulfure de carbone.

14 asphaltic cement

ciment asphaltique

Bitume, additionné ou non d'un fondant, spécialement préparé, quant à la qualité et à la consistance, pour être employé immédiatement dans la confection des revêtements bitumineux.

15 asphalt mastic

mastic d'asphalte

Mélange formé d'un matériau asphaltique (liant) et de matériaux minéraux gradués (granulat).

16 bitume

asphalt

Cf. 01

17 bitume asphaltique

asphaltic bitumen

Cf. 13

18 bitume asphaltique raffiné

refined asphaltic bitumen

Cf. 76

19 bitumen

bitume

Mélange d'hydrocarbures naturels ou de leurs combinaisons (souvent accompagnés de leurs dérivés non métalliques) pouvant être gazeux, liquides, semi-solides ou solides et qui sont entièrement solubles dans le sulfure de carbone.

20 bitume naturel

native bitumen

Cf. 66

21 bitumineux

bituminous

Cf. 22

22 bituminous

bitumineux

Contenant du bitume ou constituant une source de bitume.

23 blown petroleum

pétrole d'oxydation

pétrole soufflé

Produit semi-solide ou solide obtenu par l'action de l'air sur un bitume liquide naturel chauffé pendant l'insufflation. 
24 brai

pitch

Cf. 73

25 brai de pétrole

petroleum pitch

Cf. 72

26 brai de première distillation

straight-run pitch

Cf. 82

27 carbene

carbène

Composant du bitume dans les pétroles, produits pétrolifères, malthes, ciments asphaltiques et bitumes naturels solides, soluble dans le sulfure de carbone mais insoluble dans le tétrachlorure de carbone.

28 carbène

carbene
Cf. 27

29 carbone fixe

fixed carbon

Cf. 44

30 carbone libre (dans les goudrons)

free carbon (in tars)

Cf. 50

31 ciment asphaltique

asphaltic cement

Cf. 14

32 coal tar

goudron de houille

Mélange de produits de distillation d'hydrocarbures, généralement de séries non saturées, obtenus dans la destruction de la houille par distillation.

33 Coke-oven tar

goudron de four à coke

Sous-produit de la fabrication du coke au moyen de charbon bitumineux.

34 compressed asphalt

asphalte comprimé

Asphalte pulvérisé, chauffé et pilonné.

35 crude asphalt

asphalte brut

Asphalte tel qu'il provient des points de production. 
36 cut-back products

produits de coupage

Résidus de pétrole ou de goudron rendus fluides par l'addition de produits de distillation.

37 dead oils

huiles lourdes

Huiles plus denses que l'eau, résultant de la distillation des goudrons.

38 dehydrated tar

goudron déshydraté

Goudron complètement débarrassé d'eau.

39 emulsion

émulsion

Mixture obtenue en mettant en suspension dans un liquide un autre liquide à l'état d'extrême division.

40 émulsion

emulsion

Cf. 39

41 farine

filler

$$
\text { Cf. } 42
$$

42 filler

farine filler

Matière finement pulvérulente ou diluant inerte, que l'on mélange aux liants bitumineux pour leur donner du corps. Les éléments impalpables sont dénommés « fleur».

43 filler

filler
Cf. 42

44 fixed carbon

carbone fixe

Matières organiques formant le résidu d'une distillation opérée à l'abri de l'air et dans des conditions déterminées de chauffe et de forme des appareils.

45 fleur

flour

Cf. 46

46 flour

fleur

Éléments impalpables d'une farine. 
47 fluidifiant

flux

Cf. 48

48 flux

fluidifiant

flux

Bitumes, généralement liquides, destinés à être associés à des bitumes plus durs, en vue de les ramollir.

49 flux

flux

Cf. 48

50 free carbon (in tars)

carbone libre (dans les goudrons)

Matières organiques contenues dans le goudron, le brai, etc., insolubles dans un liquide spécifié tel que le benzol, le toluène, le sulfure de carbone, le pyridine, etc.

51 gas house coal tar

goudron de houille d'usine à gaz

Goudron fourni par les cornues d'usines à gaz, dans la fabrication du gaz d'éclairage au moyen de charbon bitumineux.

52 goudron

$\operatorname{tar}$

Cf. 83

53 goudron de four à coke coke-oven tar

Cf. 33

54 goudron de gaz à l'eau

water-gas tar

Cf. 85

55 goudron de gaz d'huile

oil-gas tar

Cf. 67

56 goudron de houille

coal tar

Cf. 32

57 goudron de houille d'usine à gaz

gas house coal tar

Cf. 51

58 goudron déshydraté

dehydrated tar

Cf. 38 
59 goudron raffiné

refined tar

Cf. 77

60 huiles lourdes

dead oils

Cf. 37

61 malthene

malthène

Constituant du bitume, soluble dans le sulfure de carbone, dans le tétrachlorure de carbone et dans l'huile de naphte.

62 malthène

malthene

Cf. 61

63 mastic asphalt

asphalte coulé

Mastic d'asphalte fondu et mélangé à chaud avec des criblures ou du sable et étendu sur une fondation appropriée.

64. mastic d'asphalte

asphalt mastic

Cf. 15

65 native asphalt; rock asphalt

asphalte naturel

asphalte en roche

Roche imprégnée de bitume

66 native bitumen

bitume naturel

Bitume qu'on trouve dans la nature, contenant en suspension une proportion variable de matière minérale. Cette expression ne doit pas être appliquée aux résidus de la distillation des huiles asphaltiques.

67 oil-gas tar

goudron de gaz d'huile

Goudron obtenu par la décomposition des vapeurs d'huile à hautes températures dans la fabrication du gaz d'huile.

68 pétrole

petroleum

Cf. 71

69 pétrole d'oxydation

blown petroleum

Cf. 23 
70 pétrole soufflé

blown petroleum

Cf. 23

71 petroleum

pétrole

Bitume liquide tel qu'on le trouve dans la nature.

72 petroleum pitch

brai de pétrole

Résidu provenant de la distillation, de l'oxydation ou du craquage du pétrole. Certains brais de pétrole employés sur les routes sont des bitumes asphaltiques.

73 pitch

brai

Résidu noir ou brun foncé, solide ou demi-solide, fusible et agglomérant, restant après évaporation partielle ou distillation fractionnée des goudrons ou des produits goudronneux.

74 produits de coupage

cut-back products

Cf. 36

75 refined asphalt

asphalte raffiné

asphalte épuré

Asphalte qui a été soumis à un raffinage et qui a été amené à l'état convenable pour être employé immédiatement ou après l'addition d'un fondant.

76 refined asphaltic bitumen

bitume asphaltique raffiné

Bitume asphaltique ayant été débarrassé totalement ou partiellement de l'eau ainsi que des impuretés organiques qu'il contenait.

77 refined tar

goudron raffiné

Goudron débarrassé d'eau par évaporation ou une distillation poursuivie jusqu'à ce que le produit ait atteint la consistance désirée ; ou encore : produit obtenu en additionnant un résidu goudronneux avec un distillat goudronneux.

78 résidus de pétrole

topped petroleum

Cf. 84

79 roche asphaltique

rock asphalt (2)

Cf. 81 
80 rock asphalt (1) ; native asphalt

asphalte naturel

asphalte en roche

Cf. 65

81 rock asphalt (2)

roche asphaltique

Roche (calcaire, grès ou autre roche massive) naturellement imprégnée de bitume asphaltique.

82. straight-run pitch

brai de première distillation

Brai obtenu directement à la consistance voulue, dans la distillation, sans fluidification ultérieure.

$83 \operatorname{tar}$

goudron

Produit bitumineux, visqueux ou liquide, provenant de la destruction par distillation de matières organiques. Le mot "goudron" doit toujours être suivi du nom de la matière dont il est extrait : houille, schiste, lignite, tourbe, végétaux.

84 topped petroleum

résidus de pétrole

Pétrole débarrassé de ses constituants les plus volatils par le procédé du « topping ».

85 water-gas tar

goudron de gaz à l'eau

Goudron obtenu par la décomposition des vapeurs d'huile à hautes températures dans la fabrication du gaz à l'eau.

Henri Dumas 